\title{
The Heron parameters of a triangle
}

\author{
ALAN F. BEARDON and PAUL STEPHENSON
}

\section{The problem}

If a triangle has sides of integer lengths, and an inscribed circle of unit radius, then it is a right-angled triangle with sides of lengths 3, 4 and 5. To see that this is the only right-angled triangle with these properties draw a right-angled triangle with sides of integer lengths $a, b$ and $c$ (the hypotenuse), and inscribed circle of unit radius. Obviously $a>1$ and $b>1$, so we can write $a=1+a_{1}$ and $b=1+b_{1}$ for positive integers $a_{1}$ and $b_{1}$. As the two tangents from a point outside a circle to the circle are of equal length, we see that $c=a_{1}+b_{1}$. Thus

$$
\left(a_{1}+b_{1}\right)^{2}=c^{2}=a^{2}+b^{2}=\left(1+a_{1}\right)^{2}+\left(1+b_{1}\right)^{2},
$$

or $\left(a_{1}-1\right)\left(b_{1}-1\right)=2$; hence $\left\{a_{1}, b_{1}\right\}=\{2,3\}$ as required. However, how do we show that this is the only (not necessarily right-angled) triangle with these properties?

This paper is concerned with the following more general problem. Let $T$ be a triangle with vertices $A, B$ and $C$, sides of lengths $a, b$ and $c$ (opposite the vertices $A, B$ and $C$, respectively), and an inscribed circle (the incircle of $T$ ) of radius $r$ (the inradius of $T$ ) and centre $I$. We say that $T$ is an integral triangle if $a, b, c$ and $r$ are positive integers, and we wish to find all integral triangles with a given inradius $r$. There are many examples and discussions in the literature on this topic; for example the number of integral triangles with inradius $n, n=1,2,3, \ldots$, is discussed in The on-line encyclopedia of integer sequences [1]. There is some overlap here with the paper [2] (and no doubt with others too), and the second author has written a diary [3] which charts the progress of this paper (during which time we were unaware of [2]).

Here we shall present an account of this problem that is based on a parametrisation of the space of all triangles by parameters which we shall call the Heron parameters of a triangle. The space of triangles can be parametrised, up to congruence, by the set of lengths of the three sides of the triangle. However, the disadvantage of these parameters is that not every triple of positive numbers yields a triangle. By contrast, every triple of positive Heron parameters does produce a triangle, and this leads to simpler discussion of our problem. For example, while there is no triangle whose triple of side lengths is $(1,2,100)$, there is a triangle whose triple of side lengths is $(1+2,1+100,2+100)$. While many (perhaps most) authors have used these ideas when discussing the problem, we are suggesting here that the Heron parameters are worthy of a more prominent role in the discussion than they have hitherto been given. In particular, we shall use Heron parameters to show, perhaps surprisingly, that there is only one acute-angled integral triangle whose inradius is a given prime number. 
2. The Heron parameters of a triangle

The Heron parameters $(u, v, w)$ of $T$ are defined geometrically in Figure 1 , and analytically by the equations

$$
2 u=b+c-a, \quad 2 v=c+a-b, \quad 2 w=a+b-c,
$$

or, equivalently, by

$$
a=v+w, \quad b=w+u, \quad c=u+v .
$$

We call $(u, v, w)$ the Heron parameters because, as we shall see, they appear in Heron's formula for the area of $T$.

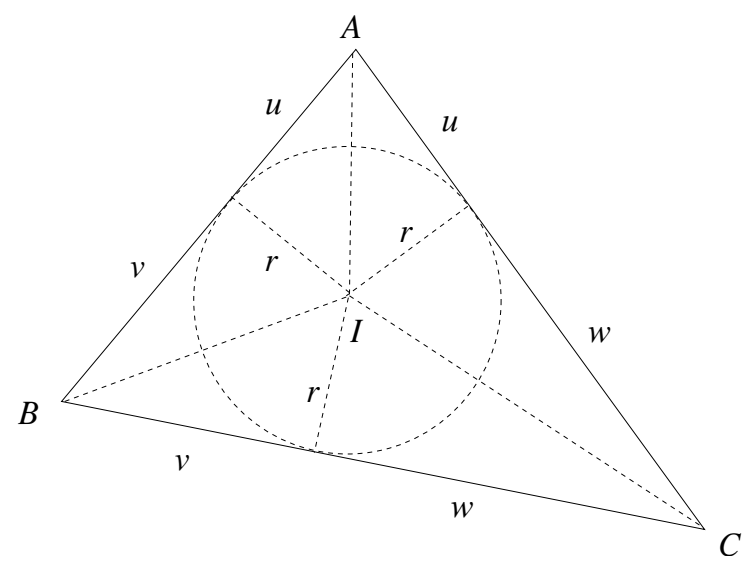

FIGURE 1: The Heron parameters of the triangle $T$

Suppose that $(u, v, w)$ is any triple of positive numbers, and we define $a, b$ and $c$ by (2). Then $a, b$ and $c$ automatically satisfy the usual triangle inequalities (for example, $c \leqslant a+b$ ), so that there does exist a triangle $T$ whose sides have lengths $a, b$ and $c$ (and which is unique up to congruence). Moreover, the Heron parameters of $T$ are $(u, v, w)$. These observations are included in the next result which shows how the Heron parameters are related to the inradius of a triangle.

Theorem 1: Let $(u, v, w)$ be any triple of positive numbers. Then there exists a triangle $T$, unique up to congruence, whose sides have lengths $v+w, w+u$ and $u+v$, whose Heron parameters are $(u, v, w)$ and whose inradius $r$ satisfies

$$
r^{2}(u+v+w)=u v w
$$

or, equivalently,

$$
\left(u v-r^{2}\right)\left(u w-r^{2}\right)=r^{2}\left(r^{2}+u^{2}\right) .
$$

Further, if $T$ is an integral triangle then $u, v$ and $w$ are integers. Finally, $T$ is obtuse-angled if $\min \{u, v, w\}<r$, right-angled if $\min \{u, v, w\}=r$, and acute-angled if $\min \{u, v, w\}>r$. 
Proof: By Heron's formula, the area of $T$ is $\sqrt{s(s-a)(s-b)(s-c)}$ or, equivalently, $\sqrt{s u v w}$, where $s=\frac{1}{2}(a+b+c)=u+v+w$. If we divide $T$ into three triangles by the segments $A I, B I$ and $C I$ we see that $T$ has area $R S$; thus $r^{2} s=u \nu w$, which is (3). It is an elementary exercise to show that (4) is equivalent (3).

Now suppose that $T$ is an integral triangle, and let $U=2 u, V=2 v$ and $W=2 w$. As $a, b$ and $c$ are integers, we see from (1) that $U, V$ and $W$ are integers. Then, from (3), $4 r^{2}(U+V+W)=U V W$ so that at least one of $U, V$ and $W$ is an even integer. However, as $U+V=2 c$, $V+W=2 a$ and $W+U=2 b$, we see that $U, V$ and $W$ have the same parity. Thus they are all even integers; hence $u, v$ and $w$ are integers. To prove the last assertion, suppose that the angles of $T$ at $A, B$ and $C$ are $2 \alpha$, $2 \beta$ and $2 \gamma$, respectively; then $u \tan \alpha=v \tan \beta=w \tan \gamma=r$, and the stated result follows from this.

We may assume that the Heron parameters of a given integral triangle $T$ satisfy $1 \leqslant u \leqslant v \leqslant w$. Then $u^{2} w \leqslant u v w=r^{2}(u+v+w) \leqslant 3 r^{2} w$, so that $1 \leqslant u \leqslant \sqrt{3} r$ (in fact, as $\sqrt{3}$ is irrational we have $u<\sqrt{3} r$ ). Thus, given $r, u \in\{1,2, \ldots, M\}$, where $M$ is the integer part of $\sqrt{3} r$. Next, for each of these values of $u$ we can write (in different ways) $r^{2}\left(r^{2}+u^{2}\right)=k_{1} k_{2}$, where $k_{j}$ are positive integers with $k_{1} \leqslant k_{2}$, and then put $v=\left(r^{2}+k_{1}\right) / u$ and $w=\left(r^{2}+k_{2}\right) / u$. These values of $v$ and $w$ satisfy (4), and this provides us with an algorithm (and a computer program) for listing all integral triangles with a given integral inradius $r$. We leave the reader to check (or consult [2]) that the only integral triangle with $r=1$ has Heron parameters $(1,2,3)$, and therefore has sides of lengths 3,4 and 5 .

In a similar way we can find all integral triangles with $r=2$. First, we have $1 \leqslant u \leqslant 3$. Then, by factorising $r^{2}\left(r^{2}+u^{2}\right)$ (which is either 20, 32 or 52), we see that $\{u, v, w\}$ is one of

$$
\{1,5,24\}, \quad\{1,6,14\}, \quad\{1,8,9\}, \quad\{2,3,10\}, \quad\{2,4,6\},
$$

and the triple $(a, b, c)$ of side lengths is one of

$$
(6,25,29), \quad(7,15,20), \quad(9,10,17), \quad(5,12,13), \quad(6,8,10) \text {. }
$$

The first three of these are obtuse-angled triangles; the last two are rightangled triangles. Finally, for $r=3,4,5, \ldots$ we can take $k_{1}$ to be, for example, any of $1, r$ and $r^{2}$; then $(u, v, w)$ is one of

$$
\left(1, r^{2}+1, r^{4}+2 r^{2}\right), \quad\left(1, r^{2}+r, r^{3}+r^{2}+2\right), \quad\left(1,2 r^{2}, 2 r^{2}+1\right)
$$

and these provide three obtuse-angled integral triangles with inradius $r$ for which $(a, b, c)$ is one of the triples

$$
\begin{gathered}
\left(r^{2}+2, r^{4}+2 r^{2}+1, r^{4}+3 r^{2}+1\right), \\
\left(r^{2}+r+1, r^{3}+r^{2}+r+1, r^{3}+2 r^{2}+2 r\right), \\
\left(2 r^{2}+1,2 r^{2}+2,4 r^{2}+1\right) .
\end{gathered}
$$


In each case, $2+c=a+b$, so that $\operatorname{gcd}(a, b, c)$ is 1 or 2 . As each of these triangles has a side whose length is odd we see that, in each case, $\operatorname{gcd}(a, b, c)=1$.

\section{Acute-angled integral triangles}

Throughout this section $T$ will be an acute-angled integral triangle whose sides have lengths $a, b$ and $c$, whose Heron parameters are $u, v$ and $w$, and whose inradius is $r$. We may assume that $u \leqslant v \leqslant w$; thus from Theorem 1,

$$
r<u \leqslant v \leqslant w .
$$

Next, from (3) we obtain

$$
\frac{1}{r^{2}}=\frac{1}{u v}+\frac{1}{v w}+\frac{1}{u w} .
$$

This shows that $\frac{1}{r^{2}} \leqslant \frac{3}{u v}<\frac{3}{r v}$ so that $v<3 r$. In addition, as $u \geqslant r+1$ and $v \geqslant r+1$, we have

$$
\frac{1}{r^{2}} \leqslant \frac{1}{(r+1)^{2}}+\frac{2}{(r+1) w}
$$

and, as $r \geqslant 1$, this simplifies to give $w \leqslant 4 r^{2} / 3$. In conclusion, we have seen above that $u<\sqrt{3} r$, so that if $T$ is an acute-angled integral triangle then, in addition to (6), we have

$$
u<\sqrt{3} r, \quad v<3 r, \quad w \leqslant \frac{4 r^{2}}{3} .
$$

This provides a reasonably efficient way to find all acute-angled integral triangles with a given inradius $r$. We simply check whether, for $u=r+1, \ldots, M$ (recall that $M$ is the integer part of $\sqrt{3} r$ ) and $v=u, \ldots, 3 r-1$, the value of $w$, where $w=\frac{r^{2}(u+v)}{u v-r^{2}}$, is an integer. If it is then $(u, v, w)$ provides such a triangle, and all such triangles will be obtained in this way.

In his recent paper [2] Bob Burn asked for all acute-angled integral triangles whose inradius is prime. He found one such triangle, say $T_{0}$, with side lengths $(10,10,12)$ and inradius 3 , and stated that he had found no others with a prime inradius at most 97 . We shall now show that $T_{0}$ is the only acute-angled integral triangle whose inradius is a prime. Let $T$ be an integral triangle whose inradius $r$ is prime. From (3), $r^{2}$ divides $u v w$ and, as $r<u<\sqrt{3} r<2 r$, we see that $r$ does not divide $u$. Thus $r^{2}$ divides $v w$, and, as $r$ is prime, one of the following cases must arise: (i) $r^{2}$ divides $v$; (ii) $r^{2}$ divides $w$; (iii) $r$ divides both $v$ and $w$. Note that in Cases (i) and (iii), $r$ divides $v$ so that, from (8), $v=2 r$.

In Case (i), $v=2 r$ and, as $r^{2}$ divides $v$, we see that $r=2$. We know 
all integral triangles with $r=2$, and none is acute-angled; thus (i) does not arise.

In Case (ii), $r^{2}$ divides $w$ and so, from (3), $w=r^{2}$. Thus $r^{2}\left(u+v+r^{2}\right)=u v r^{2}$, which simplifies to $(u-1)(v-1)=1+r^{2}$. However, $r+1 \leqslant u \leqslant v$ and, as we cannot have $u=v=r+1$, we must have $(u-1)(v-1) \geqslant r(r+1)>1+r^{2}$; thus Case (ii) does not arise.

We have now proved that Case (iii) occurs; that is, $r$ divides both $v$ and $w$. In this case, $v=2 r$ and $w=k r$, where $k$ is some integer and $k \geqslant 2$. Thus, from (3), $u=\frac{r(2+k)}{2 k-1}$ and, since $u>r$, we obtain $k=2$. This gives $u=4 r / 3$ and $v=w=2 r$. As $u$ is an integer and $r$ is prime, we have $r=3, u=4$ and $v=w=6$. This shows that $T_{0}$ is the only integral triangle with a prime inradius.

\section{The parametrisation of Pythagorean triangles}

We say that $T$ is a Pythagorean triangle if it is a right-angled triangle whose sides have integral lengths $a, b$ and $c$, and that it is a primitive Pythagorean triangle if, in addition, $\operatorname{gcd}(a, b, c)=1$. It was known, at least as early as 1894 (see [4]), that the inradius of a Pythagorean triangle is an integer; for a more recent proof (which uses Lemma 4.1) see [5, p. 288]. In any event, this shows that a Pythagorean triangle is an integral triangle.

We now recall the following well known parametrisation of primitive Pythagorean triangles; see, for example, [5, p. 285].

Theorem 2: Let $a, b$ and $c$ be positive integers such that $a^{2}+b^{2}=c^{2}$, and $\operatorname{gcd}(a, b, c)=1$. Then $a$ and $b$ are of different parity. If we relabel (if necessary) so that $a$ is even and $b$ is odd, then there are positive, coprime integers $p$ and $q$, of opposite parity and with $1 \leqslant p<q$, such that $a=2 p q, b=q^{2}-p^{2}$ and $c=q^{2}+p^{2}$.

If we use (1) to rewrite Theorem 2 in terms of the Heron parameters, we obtain the following result; see Figure 2.

Theorem 3: Let $T$ be the primitive Pythagorean triangle with parameters $p$ and $q$ as given in Theorem 2. Then the Heron parameters of $T$ are (in some order $)(q(q-p), p(q+p), p(q-p))$.

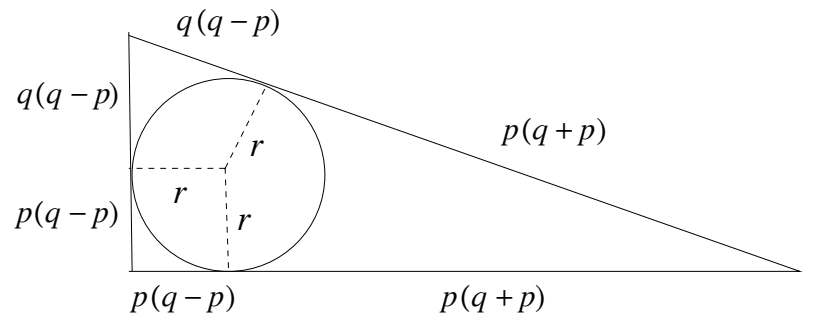

FIGURE 2: The Heron parameters of a primitive Pythagorean triangle 
We also mention that there is another, less well known, parametrisation of primitive Pythagorean triangles which appears in [4] (published in 1894, but see [6]).

Theorem 4: Let $a, b$ and $c$ be positive integers such that $a^{2}+b^{2}=c^{2}$, and $\operatorname{gcd}(a, b, c)=1$. Then there are coprime integers $m$ and $n$ such that $\sqrt{2 m n}$ is an even integer, say $2 k$, and $(a, b, c)=(m+2 k, n+2 k, m+n+2 k)$.

We shall call the parameters $m, n$ and $k$ in Theorem 4 the Dickson parameters and, in contrast to the parameters $p$ and $q$ in Theorem 2, the side lengths $a$ and $b$ and $c$ are linear functions of these parameters. As the same is true of the Heron parameters, there must be a linear relation between the Dickson parameters and the Heron parameters. There is, and the Heron parameters of the triangle are $(n+k, m+k, k)$. As the Dickson parameters are only available for Pythagorean triangles, and as they are linearly related to the Heron parameters, we may reasonably regard the Heron parameters as a generalisation of the Dickson parameters.

\section{Pythagorean triangles with a given inradius}

We shall now give a formula for the number of Pythagorean triangles, and for the number of primitive Pythagorean triangles, that have a given inradius $r$.

Theorem 5: Let $r$ be a positive integer. Then the number $P(r)$ of Pythagorean triangles with inradius $r$ is $\frac{1}{2} d\left(2 r^{2}\right)$, where $d(n)$ is the number of positive divisors of $n$. Further, the number $P_{0}(r)$ of primitive Pythagorean triangles with inradius $r$ is the number of odd unitary divisors of $r$ (that is, the number of positive, odd divisors $m$ of $r$ such that $\operatorname{gcd}(m, r / m)=1)$.

The function $d(n)$ (which is often denoted by $\tau(n)$ in the literature) is discussed at some length in [7, pp. 260-263]. As each positive divisor of $n$ lies in $\{1, \ldots, n\}$, we see that $d(n) \leqslant n$. In fact, by a much deeper argument it is shown in [7] that, for any positive $\varepsilon, \frac{d(n)}{n^{\varepsilon}} \rightarrow 0$ as $n \rightarrow \infty$. This implies that, for any positive $\varepsilon, \frac{P(r)}{r^{\varepsilon}} \rightarrow 0$ as $r \rightarrow \infty$. Thus, ultimately, $P(r)$ is less than any positive power of $r$.

Proof of Theorem 5: There is a 1-1 correspondence between integral triangles and their triples of Heron parameters $(u, v, w)$ with $u \leqslant v \leqslant w$; thus the problem is to count those triples of Heron parameters that correspond to integral Pythagorean triangles with inradius $r$. For such triangles, $u=r$ and, from (4), $(v-r)(w-r)=2 r^{2}$. As $2 r^{2}$ is not the square of an integer, $v \neq w$ so that $v-r<w-r$. It follows that the 
number of possible choices of $v$ is the same as the number of divisors $k$ of $2 r^{2}$ such that $k<2 r^{2} / k$. This number is $\frac{1}{2} d\left(2 r^{2}\right)$ and, as each $v$ determines the value of $w, P(r)=\frac{1}{2} d\left(2 r^{2}\right)$.

Each primitive Pythagorean triangle with sides of lengths $a, b$ and $c$, where $a^{2}+b^{2}=c^{2}$ and $a$ is even, produces a pair of positive coprime integers $p$ and $q$ of different parity, and with $p<q$, as given in Theorem 2 . As $p$ and $q$ are uniquely determined by $a, b$ and $c$, and each such pair of $p$ and $q$ produces a triangle, we see from Theorem 3 that the number of integral primitive Pythagorean triangles with inradius $r$ is the number of ways we can write $r=p(q-p)$, where $p$ and $q-p$ are coprime (as $p$ and $q$ are), and $q-p$ is odd (as $p$ and $q$ have different parities). The given formula for $P_{0}(r)$ follows from this.

There are many examples in [1], and we have provided all of the information needed to obtain these. Here we give one more example. We have seen that there is only one primitive Pythagorean triangle with $r=1$, and also when $r=2$. Now suppose that $r$ is a prime, and $r \geqslant 3$. Then, starting with either of the Heron parameters

$$
\left(r, r+1,2 r^{2}+r\right),\left(r, r+2, r^{2}+r\right),
$$

we obtain the two Pythagorean triangles whose triple of side lengths is one of the triples

$$
\left(2 r+1,2 r^{2}+2 r, 2 r^{2}+2 r+1\right) \quad\left(2 r+2, r^{2}+2 r, r^{2}+2 r+2\right) .
$$

We leave the reader to check that each of these is a primitive Pythagorean triangle; thus for any prime $r$ with $r \geqslant 3$ there are at least two primitive Pythagorean triangles with inradius $r$.

\section{References}

1. N. J. A. Sloane, The on-line encyclopedia of integer sequences, accessed February 2015 at http://oeis.org/A120062.

2. R. P. Burn, Faux Triangles, Mathematics Teaching, MT 241 (July 2014).

3. P. Stephenson, Alan Beardon's Problem, Mathematics Teaching, MT 241 (July 2014), electronic supplement MT Extra (5).

4. L. E. Dickson, Lowest integers representing sides of a right triangle, Amer. Math. Monthly 1 (1894) pp. 6-11.

5. D. M. Burton, Elementary number theory (2nd edn.), Wm. C. Brown, Dubuque, Iowa (1989).

6. L. J. Gerstein, Pythagorean triples and inner products, Math. Mag. 78 (2005) pp. 205-213.

7. G. H. Hardy and E. M. Wright, An introduction to the theory of numbers (5th edn.), Clarendon Press, Oxford (1979). 
doi:10.1017/mag.2015.27

ALAN F. BEARDON

Centre for Mathematical Sciences, University of Cambridge, Wilberforce Road, Cambridge CB3 OWB

e-mail:afb@dpmms.cam.ac.uk

PAUL STEPHENSON

Böhmerstraße 66, 45144, Essen, Germany e-mail: stephenson-mathcircus@t-online.de

Nemo (continued from page 204)

This month we turn the focus onto poems about spirals. The quotations are to be identified by reference to author and work. Solutions are invited to the Editor by 30th September 2015.

1. And how will your night dances

Lose themselves.

In mathematics?

Such pure leaps and spirals -

2. Year after year beheld the silent toil

That spread his lustrous coil;

Still, as the spiral grew,

He left the past year's dwelling for the new,

Stole with soft step its shining archway through,

Built up its idle door,

Stretched in his last-found home, and knew the old no more.

3. Stairs fly as straight as hawks;

Or else in spirals, curve out of curve, pausing

At a ledge to poise their wings before relaunching.

4. As up he wings the spiral stair,

A song of light, and pierces air

With fountain ardour, fountain play,

To reach the shining tops of day,

And drink in everything discern'd

An ecstasy to music turn'd

(Continued on page 242) 